\title{
Contextual variability of ADHD symptoms: embracement not erasement of a key moderating factor
}

\author{
Nanda Rommelse $\cdot$ Tessa Bunte $\cdot$ Walter Matthys $\cdot$ \\ Erica Anderson · Jan Buitelaar · Lauren Wakschlag
}

Published online: 23 December 2014

(C) Springer-Verlag Berlin Heidelberg 2014

\begin{abstract}
'Several inattentive or hyperactive-impulsive symptoms are present in two or more settings (e.g., at home, school or work; with friends or relatives; in other activities).' (p. 60, DSM-5). It is remarkable that the DSM-5 stresses symptoms rather than impairment in relation to different contexts in the diagnostic criteria for Attention-Deficit/Hyperactivity Disorder (ADHD). One would expect that pervasiveness (criterion C) means the presence of impairment of functioning, due to ADHD symptoms, in two or more settings. According to the DSM-5, a diagnosis of ADHD is warranted-when all other criteria are met-if only two out of the minimal six symptoms occur at school, even though
\end{abstract}

N. Rommelse $(\bowtie)$

Department of Psychiatry, Donders Institute for Brain, Cognition and Behavior, Radboud University Nijmegen Medical Center, Nijmegen, The Netherlands

e-mail: Nanda.Lambregts-Rommelse@ radboudumc.nl

N. Rommelse $\cdot$ J. Buitelaar

Karakter, Child and Adolescent Psychiatry University Center Nijmegen, Nijmegen, The Netherlands

T. Bunte $\cdot$ W. Matthys

Department of Psychiatry and Brain Center Rudolf Magnus,

University Medical Center Utrecht, Utrecht, The Netherlands

T. Bunte $\cdot$ W. Matthys

Department of Child and Adolescent Studies, Utrecht University, Utrecht, The Netherlands

E. Anderson $\cdot$ L. Wakschlag

Department of Medical Social Sciences and Institute for Policy

Research, Feinberg School of Medicine, Northwestern

University, Evanston, USA

J. Buitelaar

Department of Cognitive Neuroscience, Donders Institute

for Brain, Cognition and Behavior, Nijmegen, The Netherlands this is clearly below the clinical cut-off. Is a child with such a clinical profile not meaningfully different from a child exhibiting six or more symptoms in all settings? It has long been recognized that this is the case [22]. We argue that this vaguely defined criterion reflects a broader neglected issue of variability in number and contextual (in)stability of symptoms that is so typical in ADHD affected populations. We believe that variation in number and contextual expression of symptoms is a key factor to improve diagnostic and treatment procedures. Below we provide a new perspective on this issue and how to embrace and not erase it in clinical practice and research [9]. We further believe that observational assessment that allows for standardized assessment of cross-contextual variation in child behavior of the child may aid in a more precise measurement of contextual variability of ADHD symptoms in a manner that is clinically feasible and ecologically valid [7]. Observational assessment should be part of the assessment of ADHD for clinical and research purposes in a similar manner as is currently the gold standard for autism spectrum disorder (ASD).

In comparison to the DSM-IV, the DSM-5 has slightly toned down the contextual variability of symptoms. In the DSM-IV, the contextual variability of symptoms was clearly emphasized: it is very unusual for an individual to display the same level of dysfunction in all settings or within the same setting at all times (p. 81). In contrast, in the DSM-5 this contextual variability is formulated as 'typically, symptoms vary depending on context within a given setting' (p. 61). However, in both DSM versions, several situations are specifically described that suppress ADHD symptoms in individuals with ADHD, namely: when (1) frequent rewards are given for appropriate behavior (DSMIV and DSM-5); (2) the person is under very strict control (DSM-IV) or under close supervision (DSM-5); (3) the person is engaged in especially interesting activities 
(DSM-IV and DSM-5); (4) the person has consistent external stimulation (e.g., via electronic screens) (DSM-5); (5) the person is in a novel setting (DSM-IV and DSM-5); (6) the person is interacting in one-on-one situations (DSM-IV and DSM-5). Particularly the last two situations (absence of symptoms in a novel setting and in a one-on-one situation) have been used as justification for not taking into account the absence of symptoms in the clinician's office in the diagnostic process.

We completely agree with the views that ADHD symptoms may vary depending on the context that a child may still be clinically impaired when symptoms occur predominantly in one situation and less so in another [4] and also agree with the view that the absence of ADHD symptoms in the first or second clinical evaluation when a child is not challenged does not necessarily imply the absence of ADHD in this child $[3,26]$. However, we do believe that children that do not show any (or only minimal) symptoms in these first encounters-or that show symptoms predominantly in one setting and much less so in anotherare meaningfully different from children that show many symptoms across settings. Many parents tell the clinician that their child is able to keep him/herself together at school, but falls apart at home. Or the reverse, parents very reluctantly sign their child up for clinical evaluation only because the teacher urged the parents to do so, not because they themselves observed any difficulties. Both situations frequently encountered by the clinician are quite different from the clearer clinical picture where parental and teacher ratings converge. The usual unsatisfying solutions for these discrepant ratings are to (1) average the ratings [23], (2) use the teacher ratings as basis (e.g. [16], (3) use the (conservative) lowest rating as basis (often done in clinical practice), (4) analyze the ratings separately (e.g. [11]), or (5) 'add' the symptoms to a total amount and ignore in what situation these were reported (e.g. [2]). In a more accurate approach, observations in the school [1] or home situation [20] are made to complement these initial discrepant ratings. The false underlying assumption for these approaches is that discrepant ratings reflect measurement error [13] in relation to the one 'core underlying ADHD factor' present in the child: the situation may change, but the child doesn't. However, these approaches seem to ignore the fact that ADHD symptom ratings made in different situations (or in the same situation by different raters) only to a small to moderate degree reflect the same 'underlying ADHD factor' $[12,18,19]$. Merging these different sets of information into one global average, instead of adopting a multitrait-multimethod approach [14], results in an inaccurate and incomplete symptom description of the child. In fact, there is a substantial body of evidence suggesting that these informant discrepancies provide meaningful information about true variation in the child's regulation of attention and impulses depending on the social and demand characteristics of the context [8]. Different scores by different raters are therefore very informative because of differences between the contexts, different roles of the raters (parents versus teachers) and different normative schemes for the raters (teachers have more experience with age-appropriate norms). Children displaying a higher number of symptoms across settings are at increased risk for a more chronic course of the disorder [6].

When acknowledging that ADHD symptoms are-just like symptoms of Disruptive Behavior Disorder (DBD) - to a large part context dependent $[9,10]$, it is essential in good clinical practice to standardize and systematically manipulate this environmental context and to objectively quantify to what extent the ADHD symptoms are influenced by this. Observational assessment of ADHD symptoms is not part of the standard clinical routine, likely because of the assumption that ADHD symptoms can be 'masked' and a child will not show his/her 'true ADHD potential' in the first encounter. We disagree and believe that children able to 'mask' their ADHD symptoms are meaningfully different from children that are not able to do so. Recently, it was shown that ADHD symptoms can be systematically elicited and reliably observed and quantified in preschoolers with externalizing behavior problems using the Disruptive Behavior Diagnostic Observation Schedule (DB-DOS) [4, 5]. In this $1 \mathrm{~h}$ observation, the child's behavior is assessed in three interactional contexts: one parent and two examiner (engaged and non-engaged) contexts [24, 25]. The DBDOS was specifically designed to elicit high rates of clinically salient behavior in an efficient manner, in order to increase the likelihood that such variation will be observed within a standardized clinical assessment. The DB-DOS integrates observations of quality and frequency of child behavior to distinguish normative and non-normative patterns. For example, behaviors that occur in developmentally expectable contexts (e.g., difficulty inhibiting when anticipating an exciting reward) versus developmentally unexpectable context (difficulty sitting still to participate in an age-appropriate engaging activity) are distinguished. The clinical validity for ADHD has been shown to be good to excellent [4]. Of great interest is that the severity of ADHD symptoms was moderated by context: highest in the parent-child context and lowest in the examiner engaged-child context. In this study no mapping was made regarding context dependency of ADHD symptoms and differential parent and teacher ratings, but this may be expected given previous results showing that the examiner-contexts seem to serve as a proxy for the child's behavior with a non-parental adult and the parent-context uniquely related to parentidentified disruptive behavior [7]. This is particularly interesting in light of the fact that the DB-DOS does not include a peer context (which would be clinically informative but 
not feasible), yet the examiner context seems to mirror behaviors in the school setting. This suggests the possibility that the DB-DOS may be used as an observational tool to standardize and systematically manipulate the environmental context and to objectively quantify to what extent the ADHD symptoms are influenced by this. Furthermore, there is promising but preliminary evidence that the DBDOS may be particularly useful in identifying sex differences in symptom expression. It has been shown that there are sex differences in contextual patterns of DBDs, with disruptive girls demonstrating a capacity to modulate behavior with the examiner but showing very high levels of disruptive behavior with the parent whereas disruptive boys look equally disruptive across contexts [15]. As such, the DB-DOS may help support (or not) a presumption of ADHD, generated by the information from parent and teachers. It may enhance clinical decision making (particularly in the face of informant discrepancies), provide a standardized method for contextual assessment that takes into account sex differences in symptom expression and be feasibly integrated into standard clinical practice.

Another vital gap that is filled with this observational instrument is the lack of standardized direct behavioral observation in ADHD diagnostic practice. According to the Practice Parameter for ADHD [21], "the primary purpose of the interview of the child or the adolescent is not to confirm or refute the diagnosis (...) but to identify signs or symptoms inconsistent with ADHD" (p. 900). Our clinical experience, however, suggests that many parents desire the clinician to observe the child in order to arrive at a diagnosis, as parents who consult a clinician would not accept that the diagnostic decision is merely based on parent and teacher reports; parents want clinicians to "look at their child" themselves [4]. While parents are the best informants about historical features of their child's behavior, they are less skillful at making qualitative judgments about their children's behavior [25]. This is amplified during early childhood in particular when the presence of normative misbehaviors (such as impulsivity) per se is not pathognomonic. Therefore, direct observation that is not filtered through the perceptions of the parent may provide a different window on the child's functioning [17]. Moreover, we recommend that this observation is carried out without any prior knowledge regarding the child's history or clinical file. In this way, completely independent information is gathered and an unbiased measurement can be added to the diagnostic process. This may greatly help parents in the process of accepting the derived diagnosis for their child and answers to the sometimes strong critique in public media regarding the subjectivity of the ADHD diagnosis. Additional research in large samples of children at both preschool and school age, and followed over time with multi-informant data on ADHD severity and course will enhance the scientific knowledge about the clinical application of this approach. Although currently structured for research use (e.g. administration and coding are done by independent teams), adaptation for live coding in clinical use is underway.

In conclusion, ADHD is not at all context insensitive. Viewing ADHD as a strongly genetically determined disorder may have falsely generated the belief that the disorder must be context independent and rater differences are best viewed as measurement error. Rather, it may be the case that high contextual variability itself has strong genetic underpinnings and may differ according to sex. Instead of ignoring or erasing variation in number and contextual expression of symptoms, we believe there is a persuasive case for accounting for this variation in diagnostic practice. A blinded observation with a systematic manipulation of the context, such as the DB-DOS, provides a means for this purpose and also aids in the need of parents to have their child evaluated independently from parent and teacher's assessments. We believe that children that do not show any (or only minimal) symptoms in this assessment are meaningfully different from children that show many symptoms. The first group of children seems to have more capacity to (temporarily) inhibit the symptoms and to display developmentally appropriate behavior. This potential may be further stimulated in treatment (e.g., cognitive training) and may prove to be a protective factor for further development. Amplification of certain environmental moderators (classroom, parental behaviors) that suppress symptoms may be the first treatment of choice for these children. In contrast, the second group for whom symptoms are even clearly present in novel and one-on-one situations may potentially be more severely affected and may need a more rigorous approach towards treatment (e.g., medication). Thus, the variation in number and contextual expression of symptoms in the home, school and observational assessment is a crucial piece of information in the diagnostic process that is now often neglected, but may valuably contribute to clinical decision making. Especially a blinded observation without knowledge of the existing patient file would contribute as a third source of independent information and will help the parents in their process of accepting the diagnosis.

\section{References}

1. Abikoff HB, Jensen PS, Arnold LL, Hoza B, Hechtman L, Pollack S et al (2002) Observed classroom behavior of children with ADHD: relationship to gender and comorbidity. J Abnorm Child Psychol 30(4):349-359

2. Brookes K, Xu X, Chen W, Zhou K, Neale B, Lowe N et al (2006) The analysis of 51 genes in DSM-IV combined type attention deficit hyperactivity disorder: association signals in DRD4, DAT1 and 16 other genes. Mol Psychiatry 11(10):934-953 
3. Buitelaar JK, Swinkels SH, de Vries H, van der Gaag RJ, van Hooff JA (1994) An ethological study on behavioural differences between hyperactive, aggressive, combined hyperactive/ aggressive and control children. J Child Psychol Psychiatry 35(8):1437-1446

4. Bunte TL, Laschen S, Schoemaker K, Hessen DJ, van der Heijden PG, Matthys W (2013) Clinical usefulness of observational assessment in the diagnosis of DBD and ADHD in preschoolers. J Clin Child Adolesc Psychol 42(6):749-761

5. Bunte TL, Schoemaker K, Hessen DJ, van der Heijden PGM, Matthys W (2013) Clinical usefulness of the kiddie-disruptive behavior disorder schedule in the diagnosis of DBD and ADHD in preschool children. J Abnorm Child Psychol 41:681-690

6. Bunte T, Schoemaker K, Hessen DJ, van der Heijden PG, Matthys W (2014) Stability and change of ODD, CD and ADHD diagnosis in referred preschool children. J Abnorm Child Psychol 42:1213-1224

7. De Los Reyes A, Henry DB, Tolan PH, Wakschlag LS (2009) Linking informant discrepancies to observed variations in young children's disruptive behavior. J Abnorm Child Psychol 37(5):637-652

8. De Los Reyes A, Kazdin AE (2005) Informant discrepancies in the assessment of childhood psychopathology: a critical review, theoretical framework, and recommendations for further study. Psychol Bull 131(4):483-509

9. Dirks MA, De Los Reyes A, Briggs-Gowan M, Cella D, Wakschlag LS (2012) Annual research review: embracing not erasing contextual variability in children's behavior-theory and utility in the selection and use of methods and informants in developmental psychopathology. J Child Psychol Psychiatry 53(5):558-574

10. Drabick DA, Gadow KD, Loney J (2007) Source-specific oppositional defiant disorder: comorbidity and risk factors in referred elementary schoolboys. J Am Acad Child Adolesc Psychiatry 46(1):92-101

11. Epstein JN, Kelleher KJ, Baum R, Brinkman WB, Peugh J, Gardner W, Lichtenstein P, Langberg J (2014) Variability in ADHD care in community-based pediatrics. Pediatrics 134(6):1136-1143

12. Freitag CM, Rohde LA, Lempp T, Romanos M (2010) Phenotypic and measurement influences on heritability estimates in childhood ADHD. Eur Child Adolesc Psychiatry 19(3):311-323

13. Gadow KD, Drabick DA (2012) Anger and irritability symptoms among youth with ODD: cross-informant versus source-exclusive syndromes. J Abnorm Child Psychol 40(7):1073-1085

14. Geiser C, Burns GL, Servera M (2014) Testing for measurement invariance and latent mean differences across methods: interesting incremental information from multitrait-multimethod studies. Front Psychol 5:1216

15. Gray SA, Carter AS, Briggs-Gowan MJ, Hill C, Danis B, Keenan K, Wakschlag LS (2012) Preschool children's observed disruptive behavior: variations across sex, interactional context, and disruptive psychopathology. J Clin Child Adolesc Psychol 41(4):499-507
16. Javo C, Rønning JA, Handegård BH, Rudmin FW (2009) Crossinformant correlations on social competence and behavioral problems in Sami and Norwegian preadolescents. Eur Child Adolesc Psychiatry 18(3):154-163

17. Le Couteur A, Gardner F (2008) Use of structured interviews and observational methods in clinical settings. In: Rutter M, Bishop D, Pine D, Scott S, Stevenson J, Taylor E, Thapar A (eds) Rutter's child and adolescent psychiatry, 5th edn. Blackwell, Oxford, pp 271-288

18. McLoughlin G, Rijsdijk F, Asherson P, Kuntsi J (2011) Parents and teachers make different contributions to a shared perspective on hyperactive-impulsive and inattentive symptoms: a multivariate analysis of parent and teacher ratings on the symptom domains of ADHD. Behav Genet 41(5):668-679

19. Merwood A, Greven CU, Price TS, Rijsdijk F, Kuntsi J, McLoughlin G, Larsson H, Asherson PJ (2013) Different heritabilities but shared etiological influences for parent, teacher and self-ratings of ADHD symptoms: an adolescent twin study. Psychol Med 43(9): 1973-1984

20. Mulligan A, Anney R, Butler L, O'Regan M, Richardson T, Tulewicz EM, Fitzgerald M, Gill M (2013) Home environment: association with hyperactivity/impulsivity in children with ADHD and their non-ADHD siblings. Child Care Health Dev 39(2):202-212

21. Pliszka S; AACAP Work Group on Quality Issues (2007) Practice parameter for the assessment and treatment of children and adolescents with attention-deficit/hyperactivity disorder. J Am Acad Child Adolesc Psychiatry 46(7):894-921

22. Schachar R, Rutter M, Smith A (1981) The characteristics of situationally and pervasively hyperactive children: implications for syndrome definition. J Child Psychol Psychiatry 22(4):375-392

23. Tripp G, Schaughency EA, Clarke B (2006) Parent and teacher rating scales in the evaluation of attention-deficit hyperactivity disorder: contribution to diagnosis and differential diagnosis in clinically referred children. J Dev Behav Pediatr 27(3):209-218

24. Wakschlag LS, Briggs-Gowan MJ, Hill C, Danis B, Leventhal BL, Keenan K, Egger HL, Cicchetti D, Burns J, Carter AS (2008) Observational assessment of preschool disruptive behavior, part II: validity of the disruptive behavior diagnostic observation schedule (DB-DOS). J Am Acad Child Adolesc Psychiatry 47(6):632-641

25. Wakschlag LS, Hill C, Carter AS, Danis B, Egger HL, Keenan K, Leventhal BL, Cicchetti D, Maskowitz K, Burns J, Briggs-Gowan MJ (2008) Observational assessment of preschool disruptive behavior, part I: reliability of the disruptive behavior diagnostic observation schedule (DB-DOS). J Am Acad Child Adolesc Psychiatry 47(6):622-631

26. Willemsen-Swinkels SH, Buitelaar JK (1996) Is the behaviour of hyperactive children disorganized and chaotic? An informationtheoretical approach. Eur Child Adolesc Psychiatry 5(3):139-146 